Dokuz Eylül Üniversitesi-Mühendislik Fakültesi Fen ve Mühendislik Dergisi

Cilt 19, Sayı 57, Eylül 2017
Dokuz Eylul University-Faculty of Engineering Journal of Science and Engineering Volume 19, Issue 57, September 2017

\title{
Knowledge Embedded Automatic Model Generation in Microwave Design Using Knowledge Based Artificial Neural Networks
} Murat SIMSEK ${ }^{*}$

${ }^{1}$ İstanbul Teknik Üniversitesi, Uçak ve Uzay Bilimleri Fakültesi, Uzay Mühendisliği Bölümü, 34469, İstanbul

(Alınış / Received: 14.07.2016, Kabul / Accepted: 31.05.2017, Online Yayınlanma / Published Online: 20.09.2017)

Keywords Computer Aided Design (CAD), Modeling, Optimization, Artificial Neural Networks, Knowledge Based Modeling, Design Automation

Abstract: Artificial neural networks have emerged as a powerful technique for RF/microwave modeling and design. Artificial neural network parameters as number of neurons, sampling data, which are necessary for training can be utilized through automatic model generation without extra effort of user and can provide an efficient model with desired accuracy. In this work, an efficient modeling strategy combining a prior knowledge with automatic model generation technique is proposed. The aim of this combination is to decrease the need for time consuming fine model response and to increase the performance of automatic model generation algorithm using coarse model during the modeling process. Automatic model generation requires less neuron and training data compared to former methods via prior knowledge input method. Spiral inductor model is considered to demonstrate both the advantages and the validity of this technique in terms of accuracy and time consumption.

\section{Mikrodalga Tasarımında Bilgi Tabanlı Yapay Sinir Ağlarını Kullanarak Bilginin Gömüldüğü Otomatik Model Üretimi}

\begin{abstract}
Anahtar Kelimeler Bilgisayar Destekli Tasarım (CAD), Modelleme, Optimizasyon, Yapay Sinir Ağları, Bilgi Tabanlı Modelleme, Tasarim Otomasyonu

Özet: Yapay sinir ă̆ları RF / mikrodalga modelleme ve tasarımı için güçlü bir teknik olarak ortaya çıkmıştır. Yapay sinir ağlarının nöron sayısı ve örnekleme verileri gibi gerekli ĕgitim parametrelerini kullanıcının ekstra çabası olmadan kullanabilen otomatik model üretimi, arzu edilen doğrulukta etkili bir model sağlayabilir. Bu çalışmada, ön bilgi ile otomatik model üretim tekniğini birleştiren verimli bir modelleme stratejisi önerilmiştir. Bu kombinasyonun amacl, zaman alan iyi model cevabına olan ihtiyacı azaltmak ve kaba modeli kullanan otomatik model üretim algoritmasinın performansinı modelleme esnasinda arttırmaktır. Otomatik model üretimi, ön bilgi giriş yöntemi sayesinde önceki yöntemlere klyasla daha az nöron ve ĕgitim verisi gerektirmektedir. Sarmal endüktans modeli, bu tekniğin doğruluk ve zaman tüketimi açısından hem avantajlarını hem de geçerliliğini kanıtlamak için hesaba katılmıştır.
\end{abstract}




\section{Introduction}

Modelling of components and devices for Radio Frequency (RF) and microwave circuits is quite significant part of Electromagnetic (EM) design and optimization that has caused several improvements in Computer-Aided Design (CAD) in the recent years [1-4]. Design optimization and 3D simulation (fine model) of RF/microwave circuits require reliable and efficient models of components and devices. The models are generally developed by 3D EM simulators which are very complex and timeconsuming as electromagnetic methods. Otherwise, simple models (coarse model) such as empirical models, equivalent circuit models and circuit simulators have less validity range and accuracy than EM simulation/measurement [5]. These models are suitable when fast model responses are needed.

In the last decades, Artificial Neural Network (ANN) techniques [5-10] have been recognized as an alternative way to create a model instead of fine model where mathematical expressions are not available. ANN structure is obtained via generating input-output pairs which are generated by the fine model. This process takes long time and also involves expert effort and knowledge. Another issue of ANN modeling is choosing structure before training process. The number of hidden neurons are needed is directly related with complexity of the fine model. The nonlinear relationship based on training set and topology is formed through ANN's variables such as initial conditions, weight coefficients and nonlinear function definitions etc. [11]. This technique is called black box optimization and modeling due to its vague internal structure. The trained ANN can be used as accurate and fast model in the design optimization [6,12-15] and CAD tools.
Since ANN gives useful model based on training set, sometimes this model is not satisfactory enough in terms of accuracy. In this case, existing knowledge about modeling problem can be incorporated with ANN structure which is called knowledge based neural network modeling. In this technique, ANN is manipulated using existing knowledge at different zones such as input (prior knowledge input method) [16,17], output (difference method) [16-18] or internal (knowledge based neural network method) $[17,19,20]$. Except them, prior knowledge input with difference method is formed by utilizing knowledge both at input and output zones [21,22]. Moreover space mapping neural network method [23-30] maps input space of fine and coarse model. Knowledge based strategy involves less training data and number of hidden neurons to obtain more accurate results than conventional ANN techniques. Considering extrapolation performance of new modeling structures, knowledge based strategy increases accuracy of extrapolation range. Extrapolation range is selected outside of training data range. While conventional ANN structure is not satisfied in this range, knowledge based ANN gives better result by the help of general characteristic of target responses which are obtained from the coarse model.

Until now, training set and number of hidden neuron have been two essential topics for ANN modeling. Using minimum data to get more accuracy has been mainly discussed in some papers $[7,11]$. Automatic Model Generation (AMG) technique was emerged to apply intelligent ANN learning process for microwave design automation [31]. In this technique, over learning and under learning detections provide adjustment of neuron and data amount during modeling. AMG also has built in simulation drivers to realize automatic data generation for adaptive data 
increment during training. To improve AMG, knowledge based neural network techniques were exploited to add available knowledge during AMG training process, so this method is called Knowledge Based Automatic Model Generation (KAMG) [32]. KAMG involves ANN model trained with coarse model data generator. Trained ANN model constitutes available knowledge in KAMG. Therefore KAMG needs less fine model data to improve accuracy than AMG.

For the first time, Knowledge Embedded Automatic Model Generation (KEM-AMG) technique that performs coarse model data generator and fine model data generator together in the same AMG algirtihm is proposed. Multi Layer Perceptron (MLP) with single hidden layer in $A M G$ is trained using prior knowledge input method. The aim of KEM-AMG technique is to gather fine model data and coarse model data to reduce necessary data and hidden neuron numbers for AMG training process. This combination ensures that the model rapidly approximates the fine model response with less training data and less hidden neurons than existing modeling techniques including AMG, thus reducing the expert effort for modeling.

\section{The Overview of Automatic Model Generation}

AMG technique has been developed to reduce expert effort during modeling process. To implement this purpose, it utilizes efficient training process to generate new training data and to add new hidden neurons when necessary. Training data consist of input and output parts. For input part, AMG generates good initial vectors from input space. Initial vectors includes all de- sign parameters (geometric variables) for detailed physical/EM simulation model. Also some frequency range are extended using frequency steps for each geometry, generates necessary input for frequency response of the specific bandwidth. After completion of input data generation, corresponding output data are generated using EM simulation model. AMG automatically generates output data through driver software included data conversion between AMG and EM simulator. It can increase training data via this adaptive sampling strategy. AMG uses two kind of mechanism such as over learning and under learning to develop new model structure. If over learning is detected during modeling AMG adds new training data. Under learning detection is involved in order to add new hidden neurons in ANN structure. Over learning and under learning detection are determined with training and validation error for each stage. At the end of the stage, AMG algorithm decides necessary mechanism to improve model accuracy. Further information about AMG algorithm can be found in [31].

KAMG technique has been developed to improve modeling efficiency by exploiting knowledge based modeling strategy. In this technique, coarse model neural network are trained by coarse model data generator. This coarse ANN structure and sub-neural model are trained by coarse model training data to satisfy initial condition for over all KAMG models. Finally using adaptive sampling for this knowledge based AMG model, over all model are trained by data obtained from fine model data generator. KAMG technique needs less fine model response than AMG, but it accomplishes more complicated process in terms of expert effort than AMG. KAMG technique contains four knowledge based methods such as, prior knowledge input, difference, knowledge based neural network and space mapping neural network. More details about this technique are followed in [32]. 
3. Proposed Knowledge Embedded AutomaticModel Generation Approach 3.1. Proposed modeling structure Previous works on automatic model generation are considered, combination of simply applicable AMG algorithm and knowledge based strategy is realized to develop considerably useful new modeling techniques. Existing knowledge about fine model are embedded in main AMG algorithm has been proposed in this work, so it is named knowledge embedded automatic model generation (KEM-AMG). KEM-AMG can use coarse and fine model to develop new model for $\mathrm{RF} /$ microwave design. This property not only provides more accurate and less complex ANN structure than AMG, but also satisfies less complicated expert operation and more automatic process than KAMG.

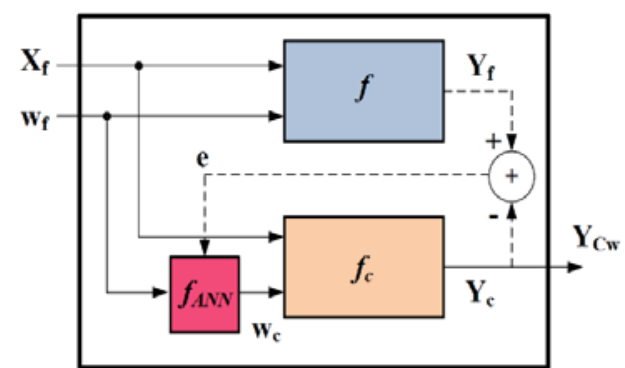

a. Space mapping via physical parameters

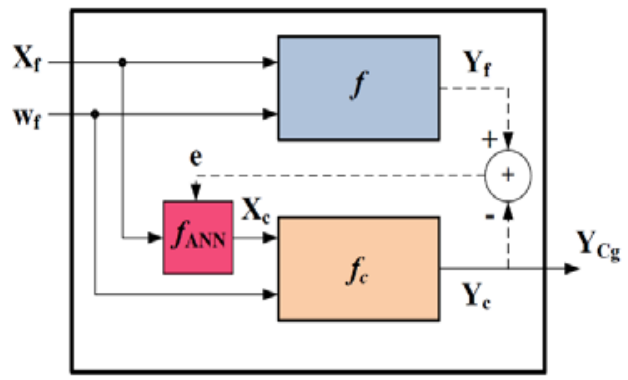

b. Space mapping via frequency

Figure 1. Coarse model improvement methods

In this approach, driver software for coarse model provides coarse model response for AMG algorithm. In addition AMG generates initial points and obtain both coarse and fine model responses for these inputs. After all generation process, AMG calculates training error and validation error for training and validation data. Existing knowledge facilitates mathematical link between input and output of the fine model through correlation between fine and coarse model responses.

Prior Knowledge Input (PKI) method has been chosen to incorporate the coarse model and the fine model in AMG modeling process. This method uses existing knowledge obtained by coarse model response as an extra input besides geometrical variables and frequency. This knowledge facilitates training process in order to form mathematical link between input and output of the fine model. Less complex relationship between input and output enables less hidden neuron usage and fast approximation for ANN. The proposed method provides these facilitates to be used by AMG algorithm without any extra expert effort.

\subsection{Coarse model improvement exploiting input mapping}

Coarse model effects the performance of knowledge based strategy. If the coarse model cannot generate approximate response comparable to the fine model, a new model cannot provide satisfactory performance according to conventional ANN. In this case, improvement of coarse model responses is necessary for knowledge based modeling application. Frequency mapping and geometrical variable mapping are probably useful way to improve coarse model as shown in Figure 1. The key idea about the improvement is creating new coarse model with limited number of fine model data.

ANN's nonlinear structure is most suitable mapping to change coarse model input space. This input mapping provides a new coarse model which is capable of 


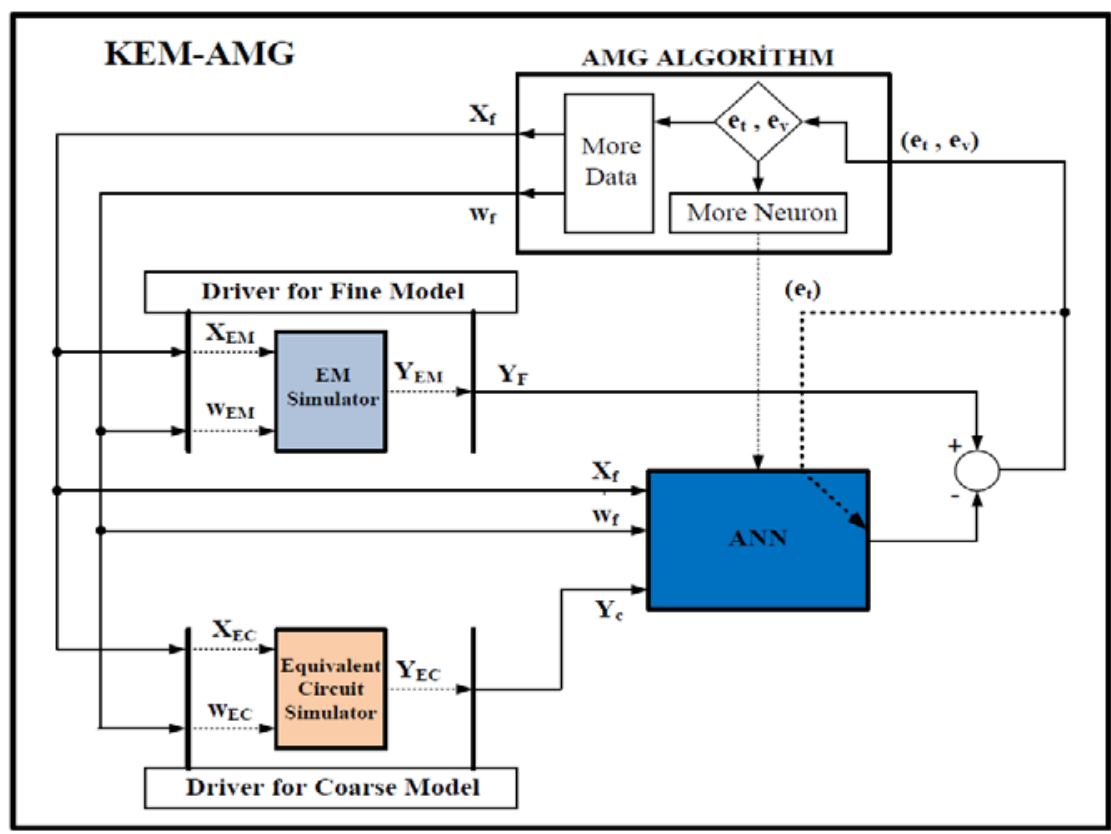

Figure 2. Knowledge embedded automatic model generation (KEM-AMG) structure

generating more approximate response of the fine model than former. However these improvements are limited because output of the fine model is not totally changed using input mapping. Knowledge based structures create output mapping except space mapping neural network. Input mapping for coarse model provides input correction and this new coarse model with knowledge based structure implements output correction for final modeling phase. Input mapping is compulsory when the dimension of the coarse model input space is different from the dimension of the fine model input space. Therefore mapping performs mathematical link between geometric variables of the fine model and component values of the equivalent circuit model. Input mapping is also utilized to obtain more approximate coarse model responses to the fine model responses.

3.3. The formulations of proposed knowledge embedded algorithm

Knowledge embedded automatic model generation technique involves the fine model responses obtained by EM simulation and the coarse model responses obtained by equivalent circuit formulation/simulator. For these simulation responses, user is supposed to create software to drive simulator, so this software tool is called driver. Driver performs necessary process in order to transform both input and output data between AMG and simulator as in Figure 2. If knowledge based model or ANN is used as a coarse model, driver for the coarse model is not necessary anymore. Because ANN models can be used in AMG algorithm without any extra process. Let $\left(x_{f} ; Y_{f}\right)$ and $\left(x_{c} ; Y_{c}\right)$ be input-output vectors of fine and coarse models, respectively. The relationship $f$ between $x_{f}$ and $Y_{f}$ can be nonlinear and multidimensional. In addition input-output relationship of the fine model is much more complex, so obtaining fine model response takes longer time than coarse model $\left(f_{c}\right)$ response. Fine and coarse model's relationships can be represented by 
$Y_{f}=f\left(x_{f}, W_{f}\right)$

$Y_{c}=f_{c}\left(x_{c}, W_{c}\right)$

where $x_{f}$ and $x_{c}$ show input and $w_{f}$ and $w_{c}$ show frequency for fine and coarse model, respectively. In proposed technique, prior knowledge input method has been chosen to approximate coarse model response to the fine model response. Coarse model input in (2) should be equal to fine model input in (1) to obtain necessary coarse model output. Input-output relation of prior knowledge input method can be represented in (3).

$$
Y_{f} \approx Y_{P K I}=f_{A N N}\left(X_{f}, W_{f}, Y_{c}\right)
$$

where $f_{A N N}$ is nonlinear function formed by ANN and $Y_{c}$ is the coarse model response for the fine model inputs. If coarse model is not accurate enough, input mapping is suitable option in order to improve the coarse model responses. After geometry mapping and frequency mapping, the responses of the coarse models are denoted in (4) and (5), respectively.

$$
\begin{aligned}
& Y_{f} \approx Y_{C g}=f_{c}(\underbrace{f_{A N N}\left(X_{f}\right)}_{x_{c}}, W_{f}) \\
& Y_{f} \approx Y_{C W}=f_{c}(X_{f}, \underbrace{f_{A N N}\left(W_{f}\right)}_{w_{c}})
\end{aligned}
$$

In (4) and (5), ANN is used to perform input mapping for geometry in Figure 1.a and frequency in Figure 1.b, respectively. In knowledge embedded automatic model generation, training set and validation set are updated during sampling stage. Sampling stage involves data generation according to validation error for each validation point in the search space. The biggest error among all of validation errors points from where more data has to be obtained in the search space. Therefore validation error is essential part for direction of data generation. KEM-AMG algorithm incorporates the coarse model response to be calculated validation error with the difference of AMG. Contribution of existing knowledge to AMG training process reduces the complexity of modeling structure thus the number of over learning and under learning detections decreases. Less detection allows less data and hidden neurons usage during KEM-AMG training process. The more accurate coarse model increases the performance of KEM-AMG and decreases necessary number of data and hidden neurons.

AMG algorithm utilizes less number of the fine model responses together with the coarse model responses in PKI method. ANN model structure for PKI is formed by adaptive data generation and adaptive hidden neuron numbers mechanism in AMG algorithm. Training error $\left(e_{t}\right)$ and validation error $\left(e_{v}\right)$ in Figure 2 are exploited to decide necessary stage for next step during optimization process of KEM-AMG. If under learning is detected during training process, KEM-AMG adds new hidden neurons to ANN structure. In case over learning is detected, more data are added to training and validation set obtained from the fine and the coarse model through drivers. KEM-AMG algorithm generates new model considering model complexity, and uses minimum hidden neuron numbers and time consuming fine model responses. Extra effort different from AMG is not necessary for proposed method during modeling. As clearly shown in Figure 2, drivers provide necessary conversion from model variables to simulator variables. KEM-AMG algorithm automatically utilized the fine and the coarse model to generate modeling data 
via this mechanism,. After data generation for each stage, all data use training of PKI model to satisfy objectives of each stage. PKI model facilitates training process via coarse model responses with respect to conventional ANN model. Training and validation error which is calculated using PKI responses and fine model responses determine objectives during optimization for each stage. Over learning and under learning detections cause more data generation and hidden neuron adjustment, respectively.

\section{Microwave Design Application: Spiral Inductor Modelling}

Spiral inductor modelling is utilized to show efficiency of proposed knowledge embedded automatic model generation technique. Spiral inductor application is selected to generate more sample and fast response to compare modeling techniques. Moreover coarse model has already developed in previous work and it is suitable to embed in AMG algorithm.

S-parameters are usually preferable for the model outputs due to ease of measurement. Since $S_{12}$ and $S_{21}$ are equal for a passive two-port reciprocal network like a spiral inductor, only $S_{12}$ is included in the model output. Here in, the Sparameters are computed using the fullwave EM simulation tools ADS Momentum from Agilent Technologies, Palo Alto, CA [33]. The structure of spiral inductor is illustrated in Figure 3, where $R_{i n}$ is the inner radius, $w$ is the width of the microstrip lines, $s$ is the spacing between lines, and $N$ is the number of turns. The substrate parameters are as follows: The Oxide substrate has relative permittivity of 3.9 and thickness of $3.97 \mu \mathrm{m}$. The Si substrate has relative permittivity of 11.9 , conductivity of 12.5 Siemens/m and thickness of $300 \mu \mathrm{m}$. The conductor layers are copper with thicknesses of $0.42 \mu \mathrm{m}$ and conductivity values of $5.8 \times 10^{7}$ Siemens/m.

This model has four input geometrical variables, i.e., $x=\left[\begin{array}{llll}N & R_{i n} & W & s\end{array}\right]^{T}$ as illustrated in Figure 3 plus frequency as an additional input of EM simulation.

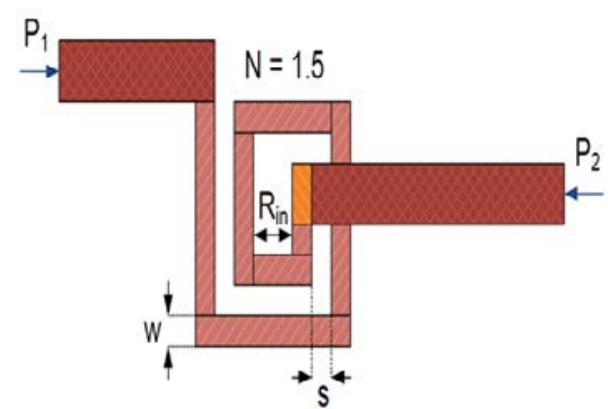

Figure 3. Physical parameters for spiral inductor design

The model has six outputs in the frequency domain, i.e., $y=\left[\begin{array}{llllll}R S_{11} & I S_{11} & R S_{12} & I S_{12} & R S_{22} & I S_{22}\end{array}\right]$

which are the real and imaginary parts of

Table 1. Data range of physical parameters for spiral inductor according to different number of data

\begin{tabular}{|c|c|c|c|c|c|c|c|c|}
\hline Data Set & \multicolumn{2}{|c|}{$\mathbf{N}$} & \multicolumn{2}{c|}{ Rin (mm) } & \multicolumn{2}{c|}{ w (mm) } & \multicolumn{2}{c|}{ s (mm) } \\
\hline & min. & max. & min. & max. & min. & max. & min. & max. \\
\hline train 800 & 1.5 & 4.5 & 30 & 125 & 5 & 23 & 1 & 10 \\
\hline train 200 & 1.5 & 4.5 & 30 & 125 & 5 & 23 & 1 & 9 \\
\hline train 50 & 1.5 & 4.5 & 30 & 120 & 5 & 23 & 1 & 9 \\
\hline train 20 & 1.5 & 4.5 & 45 & 115 & 13 & 23 & 1 & 8 \\
\hline test 100 & 1.5 & 4.5 & 35 & 125 & 5 & 23 & 2 & 10 \\
\hline adaptive & 1.5 & 2.5 & 30 & 125 & 5 & 23 & 1 & 10 \\
\hline
\end{tabular}



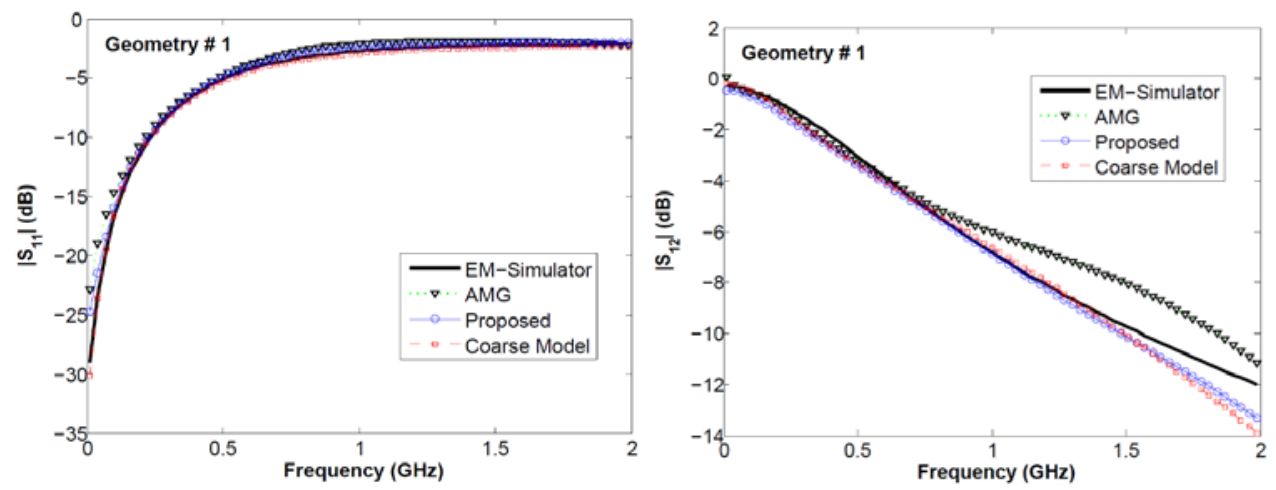

Figure 4. Comparison of S-parameters ( $S_{11}$ and $S_{12}$ ) for geometry \# 1 by this proposed KEMAMG model, the EM simulation, the coarse model(SMNN) ,and AMG model in case of 20 training data for fixed data usage
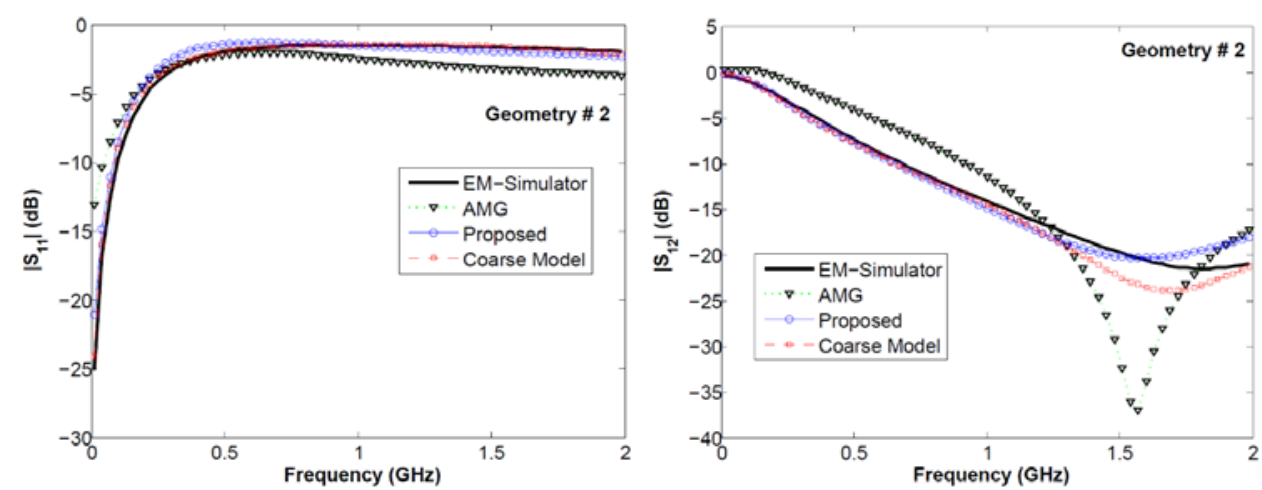

Figure 5. Comparison of S-parameters ( $S_{11}$ and $S_{12}$ ) for geometry \# 2 by this proposed KEMAMG model, the EM simulation, the coarse model(SMNN) ,and AMG model in case of 20 training data for fixed data usage

$\mathrm{S}_{11}, \mathrm{~S}_{12}$, and $\mathrm{S}_{22}$.Two different kind of data are generated for spiral inductor such as fixed data set and adaptive data set. 800 data are firstly generated by uniform distribution then other data $(200,50,20)$ are obtained from 800 data. Moreover 100 test data are generated by uniform distribution but differently than 800 data. The whole data ranges for spiral inductor are given in Table 1 . Some data range in Table 1 provide extrapolation performance for this modeling. For each geometry, the frequency range is from 10 $\mathrm{MHz}$ to $1.99 \mathrm{GHz}$ with a step size of 30 $\mathrm{MHz}$; thus 67 frequency points are used. Figure 4 - Figure 7 depict the Sparameters $\mathrm{S}_{11}, \mathrm{~S}_{12}(\mathrm{~dB})$ of the model developed by the proposed KEM-AMG method for four different spiral inductor geometries \#1, \#2, \#3 and \#4 in the testing data, and its comparison with EM data, coarse model and AMG model. The geometrical variables for different geometries are represented below:

(\#1) $\mathrm{N}=1.5, \quad$ Rin $=125 \mathrm{~mm}, \quad \mathrm{w}=5 \mathrm{~mm}$, $\mathrm{s}=10 \mathrm{~mm}$;

(\#2) $\mathrm{N}=2.5, \quad$ Rin $=125 \mathrm{~mm}, \quad w=5 \mathrm{~mm}$, $\mathrm{s}=11 \mathrm{~mm}$;

(\#3) $\mathrm{N}=3.5, \quad$ Rin $=120 \mathrm{~mm}, \quad w=7 \mathrm{~mm}$, $\mathrm{s}=10 \mathrm{~mm}$;

(\#4) $\mathrm{N}=4.5, \quad$ Rin $=70 \mathrm{~mm}, \quad \mathrm{w}=21 \mathrm{~mm}$, $\mathrm{s}=4 \mathrm{~mm}$; 
M. Simsek / Knowledge Embedded Automatic Model Generation in Microwave Design Using Knowledge Based Artificial Neural Networks
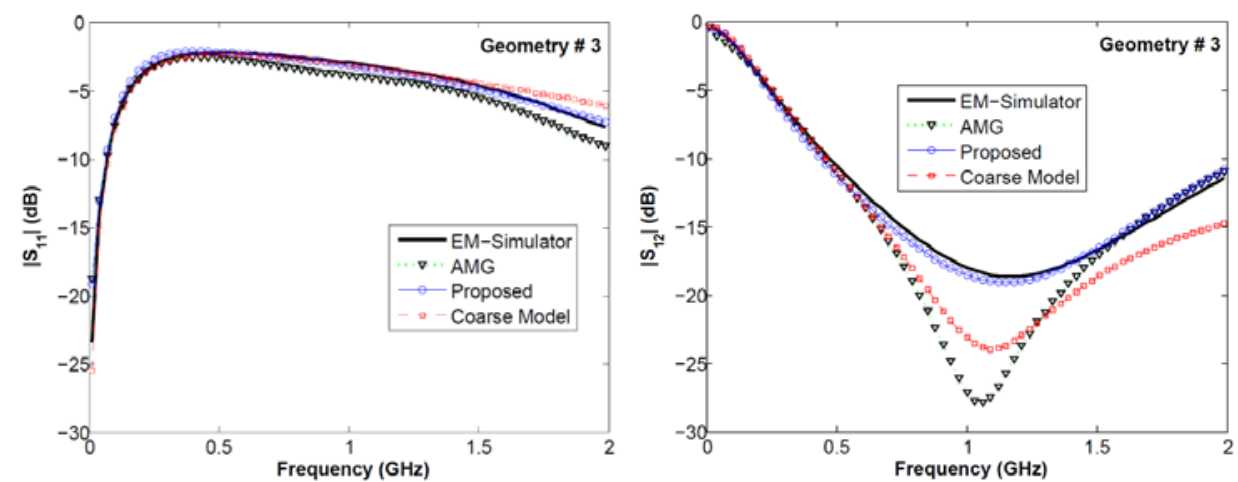

Figure 6. Comparison of S-parameters ( $\mathrm{S}_{11}$ and $\mathrm{S}_{12}$ ) for geometry \# 3 by this proposed KEMAMG model, the EM simulation, the coarse model(SMNN) , and AMG model in case of 20 training data for fixed data usage
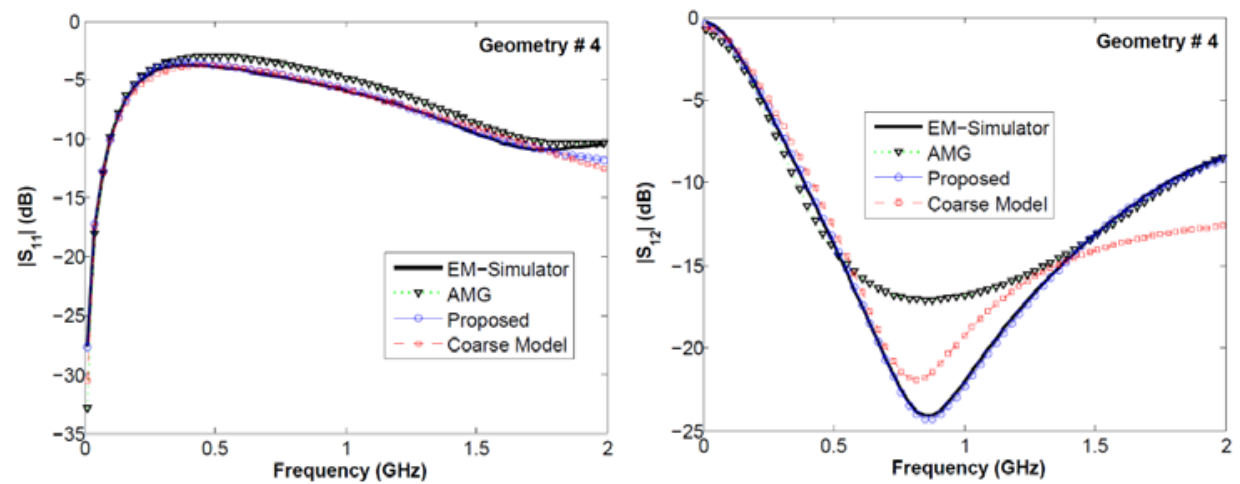

Figure 7. Comparison of S-parameters ( $S_{11}$ and $S_{12}$ ) for geometry \# 4 by this proposed KEMAMG model, the EM simulation, the coarse model(SMNN) ,and AMG model in case of 20 training data for fixed data usage

Table 2. Results of Spiral inductor for different number of data

\begin{tabular}{|c|c|c|c|c|c|c|}
\hline Data Set & \multicolumn{2}{|l|}{ Number of Neuron } & \multicolumn{2}{l|}{ Training Error (\%) } & \multicolumn{2}{l|}{ Test Error (\%) } \\
\hline & AMG & KEM-AMG & AMG & KEM-MG & AMG & KEM-AMG \\
\hline 800 & 40 & 11 & 0.648 & 0.674 & 0.639 & 0.659 \\
\hline 200 & 40 & 10 & 0.64 & 0.654 & 0.755 & 0.719 \\
\hline 50 & 32 & 9 & 0.673 & 0.676 & 1.038 & 0.821 \\
\hline 20 & 23 & 8 & 0.6402 & 0.668 & 10.812 & 1.192 \\
\hline
\end{tabular}

While selection of geometrical variables for fix data set, different kind of $\mathrm{N}$ are chosen to show output performance of spiral inductor Therefore different Sparameters are obtained to evaluate proposed method in terms of minimum training data usage. In fixed data analysis, proposed PKI-AMG and AMG methods are used to obtain training and test result in Table 2. This comparison contains 

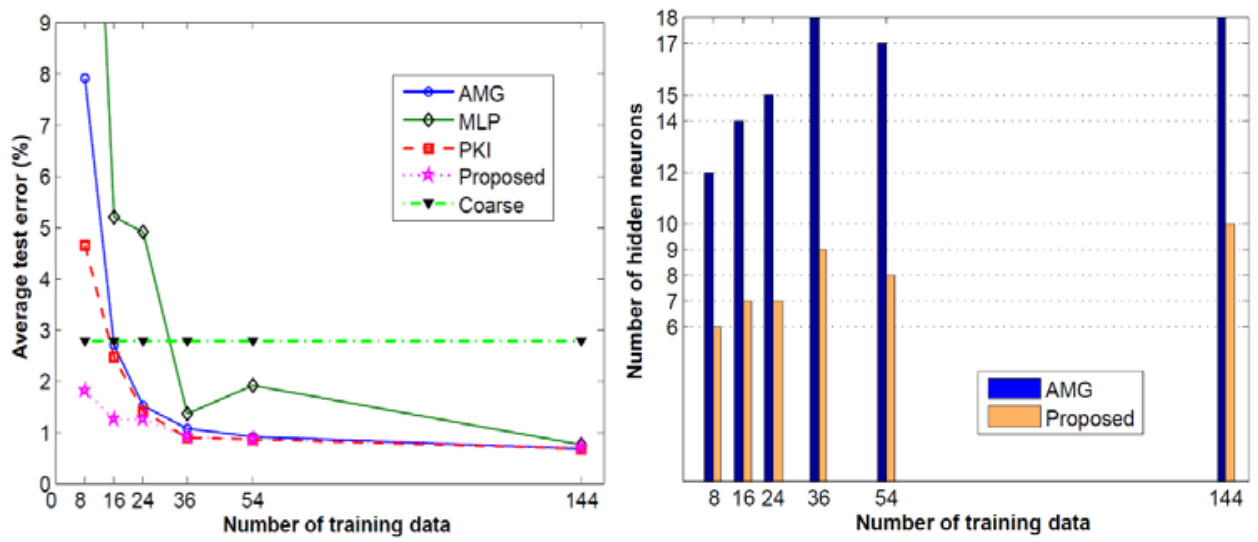

Figure 8. Comparison of average test error and hidden neuron usage in terms of training data number by this proposed KEM-AMG model, the EM simulation, the coarse model(SMNN), PKI model, MLP model ,and AMG model in case of adaptive model generation
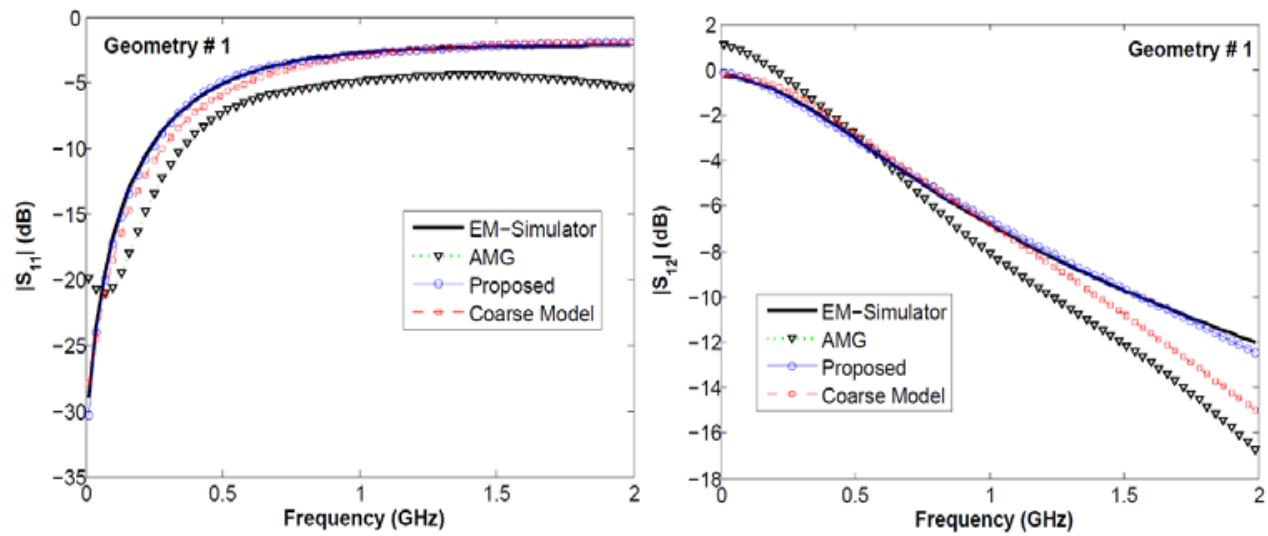

Figure 9. Comparison of S-parameters ( $S_{11}$ and $S_{12}$ ) for geometry \# 1 by this proposed KEMAMG model, the EM simulation, the coarse model(SMNN) , and AMG model in case of 8 training data for adaptive sampling stage

error analysis and hidden neuron usage for different training data set $(800,200,50,20)$. Adaptive hidden neuron number mechanism adjusts neuron numbers in hidden layer for two methods. As shown in Table 2, proposed method has less hidden neuron and better test error performance than classical AMG method. Our technique improves test result through using knowledge and AMG algorithms together as shown in Figure 8.

The coarse model is obtained by combination of space mapping neuro- model and transfer function model. This structure ensures that coarse model approximates the fine model. Adaptive sampling mechanism is used to generate new model structure, hence proposed KEM-AMG and AMG methods are compared with each other. Average test error and corresponding number of hidden neurons for each data sampling stage are shown in Figure 8. The $\mathrm{S}$-parameters $\mathrm{S}_{11}, \mathrm{~S}_{12}(\mathrm{~dB})$ of the spiral inductor geometries \#1 and \#2 (same as fixed data set) are also depicted in Figure 9 and Figure 10, respectively. In 

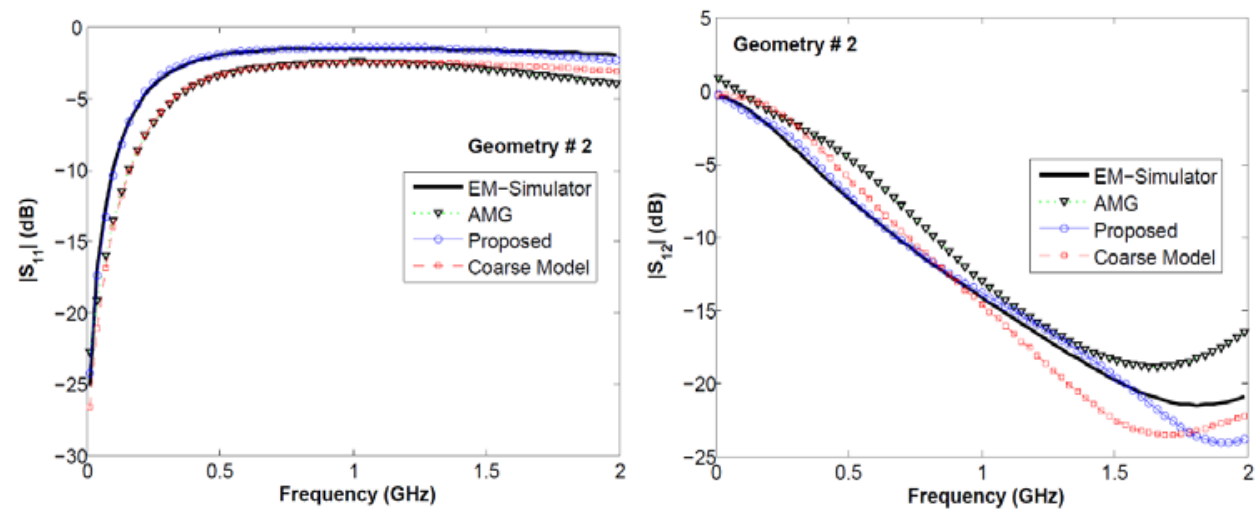

Figure 10. Comparison of S-parameters ( $S_{11}$ and $S_{12}$ ) for geometry \# 2 by this proposed KEMAMG model, the EM simulation, the coarse model(SMNN), and AMG model in case of 8 training data for adaptive sampling stage

Table 3. Results of spiral inductor for adaptive sampling

\begin{tabular}{|c|c|c|c|c|c|c|c|c|c|}
\hline \multicolumn{3}{|c|}{ Average Training Error (\%) } & \multicolumn{5}{c|}{ Average Test Error (\%) } \\
\hline $\begin{array}{c}\text { Data } \\
\text { Set }\end{array}$ & MLP & AMG & PKI & $\begin{array}{c}\text { KEM- } \\
\text { AMG }\end{array}$ & $\begin{array}{c}\text { Data } \\
\text { Set }\end{array}$ & MLP & AMG & PKI & $\begin{array}{c}\text { KEM- } \\
\text { AMG }\end{array}$ \\
\hline 144 & 0.8039 & 0.6647 & 0.7178 & 0.6767 & 154 & 0.7565 & 0.6762 & 0.6741 & 0.6892 \\
\hline 54 & 1.5835 & 0.6673 & 0.8434 & 0.6661 & 154 & 1.8955 & 0.9047 & 0.8423 & 0.8498 \\
\hline 36 & 0.8298 & 0.6676 & 0.7667 & 0.679 & 154 & 1.3587 & 1.0671 & 0.8692 & 0.9022 \\
\hline 24 & 1.3754 & 0.6672 & 0.953 & 0.6875 & 154 & 4.9076 & 1.5203 & 1.3958 & 1.2395 \\
\hline 16 & 0.9802 & 0.6776 & 0.8973 & 0.6816 & 154 & 5.2048 & 2.6928 & 2.4554 & 1.2558 \\
\hline 8 & 1.8984 & 1.4099 & 1.0942 & 0.6713 & 154 & 18.1996 & 7.8949 & 4.644 & 1.7992 \\
\hline
\end{tabular}

Table 4. Time consumption analysis of adaptive sampling according to necessary time for fine response satisfying $\% 0.65$ validation error in spiral inductor

\begin{tabular}{|c|c|c|c|c|}
\hline Method & $\begin{array}{c}\text { ANN } \\
\text { Structure }\end{array}$ & $\begin{array}{c}\text { Number of } \\
\text { Geometry }\end{array}$ & $\begin{array}{c}\text { Data Generation } \\
\text { Time per Geometry } \\
\text { (second) }\end{array}$ & $\begin{array}{c}\text { Time } \\
\text { Consumption } \\
\text { (hour) }\end{array}$ \\
\hline AMG & $(5,18,6)$ & 144 & 270 & 10.8 \\
\hline KEM-AMG & $(11,18,6)$ & 34 & 270 & 2.55 \\
\hline
\end{tabular}

adaptive data analysis, training and test result in Table 3 reveal efficiency of the proposed method. Considering different kind of data number, proposed KEM-AMG method performs better test error for the less number of data. Because knowledge based modeling is efficient way to reduce the number of training data. This consequence is obviously realized from
Table 3. Time consumption analysis in Table 4 gives satisfactory information about complexity of modeling methods and time dependent performance of the algorithms. Time is one of the most important part during modeling. Same error condition $(\% 0.65)$ is implemented for proposed KEM-AMG and AMG methods and the different number of 
geometry is obtained. According to total time consumption in Table 4, proposed method needs less time than AMG algorithm to satisfy error condition during modeling.

\section{Discussion and Conclusion}

In this paper, a novel knowledge embedded automatic model generation algorithm has been presented and applied to microwave passive component design. Knowledge based method (prior knowledge input) and automatic model generation algorithm have been utilized to perform new automatic model generation strategy. Compared to conventional automatic generation method, the proposed method has better test performance in terms of average test error, hidden neuron usage and time consumption. The most important advantage of the new technique is that fine and coarse model are embedded inside the model generation process, thus the proposed method can generate new data set for prior knowledge input structure. Therefore coarse model responses approximate fine model responses via knowledge based strategy. Knowledge embedded model decreases necessity of the number of training data. As realized in Figure 8, Adaptive sampling stage of proposed method provides more accurate results and also involves less hidden neurons than conventional methods such as SMNN, PKI, MLP and AMG through extra knowledge embedding in KEM-AMG. In addition other knowledge based methods can be applied in this method and different kind of advantages can obtain through the novel algorithm.

\section{Acknowledgement}

The author thanks to Prof. Q. J. Zhang from Carleton University for making available of spiral inductor example and also Neuro Modeler program.

\section{References}

[1] P. Burrascano, S. Fiori, and M. Mongiardo, 1999. A review artificial neural networks applications in microwave computer-aided design, Int. J. RF Microwave Computer-Aided Eng., vol. 9, no. 3, pp. 158-174. DOI: $10.1002 /($ SICI)1099047X(199905)9:3<158::AIDMMCE3>3.0.CO;2-V

[2] V. K. Devabhaktuni, M. C. E. Yagoub, F. Yang, J. Xu, and Q. J. Zhang, 2001. Computer-aided design of RF and microwave circuits and systems, Int.J. RF Microwave CAE, vol. 11, pp. 4-21. DOI: $\underline{10.1109 / 22.989983}$

[3] M. B. Steer, J. W. Bandler, and C. M. Snowden, 2002. Computer-aided design of RF and microwave circuits and systems, IEEE Trans. Microwave Theory and Tech., vol. 50, pp. 996-1005. DOI: $\underline{10.1109 / 22.989983}$

[4] V. Rizzoli, A. Costanzo, D. Masotti, A. Lipparini, and F. Mastri, 2004. Computer-aided optimization of nonlinear microwave circuits with the aid of electromagnetic simulation, IEEE Trans. Microwave Theory and Tech., vol. 52, pp. 362377.

DOI: $\underline{\text { 10.1109/TMTT.2003.820898 }}$

[5] Q. J. Zhang, K. C. Gupta, and V. K. Devabhaktuni, 2003. Artificial neural networks for $\mathrm{RF}$ and microwave design from theory to practice, IEEE Trans. Microwave Theory and Tech., vol. 51, no. 4, pp. 1339-1350.

DOI: $10.1109 /$ TMTT.2003.809179

[6] A. H. Zaabab, Q. J. Zhang, and M. S. Nakhla, 1995. Neural network modeling approach to circuit optimization and statistical design, IEEE Trans. Microwave Theory and Tech., vol. 43, pp. 1349-1358. DOI: $\underline{10.1109 / 22.390193}$ 
[7] Q. J. Zhang and K. C. Gupta, 2000. Neural networks for RF and microwave design, Boston, London: Artech House.

[8] J. E. Rayas-Sanchez, 2004. EM-based optimization of microwave circuits using artificial neural networks: the state-of-the-art, IEEE Trans. Microwave Theory and Tech., vol. 52, no. 1 , pp. 420-435. DOI: $10.1109 / T M T T .2003 .820897$

[9] X. Ding, V. K. Devabhaktuni, B. Chattaraj, M. C. E. Yagoub, M. Deo, J. $\mathrm{Xu}$, and Q. J. Zhang, 2004. Neuralnetwork approaches to electromagnetic based modeling of passive components and their applications to high frequency and high-speed nonlinear circuit optimization, IEEE Trans. Microwave Theory and Tech., vol. 52, no. 1, pp. 436-449. DOI: $10.1109 / T M T T .2003 .820889$

[10] M. Simsek, 2014. Knowledge based three-step modeling strategy exploiting artificial neural network, in Solving Computationally Expensive Engineering Problems, ser. Springer Proceedings in Mathematics Statistics, S. Koziel, L. Leifsson, and X.-S. Yang, Eds. Springer International Publishing, , vol. 97, pp. 219-239. DOI:10.1007/978-3-319-08985$\underline{0 \quad 9}$

[11] S. Haykin, 1999. Neural network - a comprehensive foundation, 2nd edition, Prentice Hall Inc., New Jersey, USA.

[12] J. Bandler, M. A. Ismail, J. E. RayasSanchez, and Q. J. Zhang, 1999. New directions in model development for $\mathrm{RF} /$ microwave components utilizing artificial neural networks and space mapping, vol. 4. Antennas and Propagation Society International Symposium, 11-16

$$
\begin{array}{lcr}
\text { July, } & \text { pp. } & 2572-2575 . \\
\text { DOI: } & \text { 10.1109/APS.1999.789334 }
\end{array}
$$

[13] M. H. Bakr, J. Bandler, M. A. Ismail, J. E. Rayas-Sanchez, and Q. J. Zhang, 2000. Neural space mapping optimization for EM-based design, IEEE Transactions on Microwave Theory and Techniques, vol. 48, no. 12, pp. 2307-2314. DOI: $10.1109 / 22.898979$

[14] H. Kabir, Y. Wang, M. Yu, and Q. J. Zhang, 2008. Neural network inverse modeling and applications to microwave filter design, IEEE Trans. Microwave Theory and Tech., vol. 56, pp. 867-879. DOI: $10.1109 /$ TMTT.2008.919078

[15] M. S. A. Aoad and Z. Aydin, 2015. Development of knowledge based response correction for a reconfigurable n-shaped microstrip antenna design, in IEEE MTT-S International Conference on Numerical Electromagnetics and Multiphysics Modeling and Optimization, 11-14 August, Ottawa,Canada. DOI:10.1109/NEM0.2015.7415078

[16] P. M. Watson, K. C. Gupta, and R. L. Mahajan, 1998. Development of knowledge based artificial neural network models for microwave components, in IEEE Int. Microwave Symp. Digest. pp. 9-12. DOI:10.1109/MWSYM.1998.68931 $\underline{2}$

[17] M. Simsek, Q. J. Zhang, H. Kabir, Y. Cao, and N. S. Sengor, 2011. The recent developments in microwave design, International Journal of Mathematical Modelling and Numerical Optimization, vol. 2, no. 2 , pp. $213 \quad$ - 228. DOI:10.1504/IJMMNO.2011.03942 9

[18] P. M. Watson and K. C. Gupta, 1996. EM-ANN models for microstrip vias 
and interconnects in dataset circuits, IEEE Trans. Microwave Theory and Tech., vol. 44, pp. 24952503. DOI: $\underline{10.1109 / 22.554584}$

[19] G. G. Towell and J. W. Shavlik, 1994. Knowledge-based artificial neural networks, Artificial Intelligence, vol. 70, pp. 119-165. DOI:10.1016/004-3702(94)90105$\underline{8}$

[20] F. Wang and Q. J. Zhang, 1997. Knowledge based neural models for microwave design, IEEE Trans. Microwave Theory and Tech., vol. 45, pp. 2333-2343. DOI: $10.1109 / 22.643839$

[21] M. Simsek and N. S. Sengor, 2008. A knowledge-based neuromodeling using space mapping technique: compound space mapping-based neuromodeling, Int. J. Numer. Model, vol. 21, no. 1-2, pp. 133-149 DOI: $10.1002 /$ inm.656

[22] M. Simsek and N. Tezel, 2012. The reconstruction of shape and impedance exploiting space mapping with inverse difference method, IEEE Transactions on Antennas and Propagation, vol. 60, no. 4, pp. 1868-1877. DOI: $10.1109 / T A P .2012 .2186225$

[23] J. W. Bandler, M. A. Ismail, J. E. Rayas-Sanchez, and Q. J. Zhang, 1999. Neuromodeling of microwave circuits exploiting space mapping technology, IEEE Trans. Microwave Theory and Tech., vol. 47, no. 12, pp. 2417-2427. DOI: $\underline{10.1109 / 22.808989}$

[24] Y. Cao and G. Wang, 2007. A wideband and scalable model of spiral inductors using spacemapping neural network, IEEE Trans. Microwave Theory and Tech., vol. 55, no. 12, pp. 24732480. DOI: $10.1109 /$ TMTT.2007.909602
[25] S. Koziel and J. W. Bandler, 2007. A space-mapping approach to microwave device modeling exploiting fuzzy systems, IEEE Trans. Microwave Theory and Tech., vol. 55, no. 12, pp. 2539 2547.

DOI: $10.1109 / T M T T .2007 .909605$

[26] S. Koziel and J. W. Bandler 2007. Microwave device modeling using space-mapping and radial basis functions, in IEEE Int. Microwave Symp. Digest. pp. 799-802, Honolulu, Hawaii. DOI: $10.1109 /$ MWSYM.2007.38007 $\underline{9}$

[27] L. Zhang and Q. J. Zhang, 2008. Neuro-space mapping technique for semiconductor device modeling, Optimization and Engineering, vol. 9, no. 4, pp. 393-405. DOI: $10.1007 / \mathrm{s} 11081-007-9024-0$

[28] L. Zhang, Q. J. Zhang, and J. Wood, 2008. Statistical neuro-space mapping technique for large signal modeling of nonlinear devices, IEEE Trans. Microwave Theory and Tech., vol. 56, pp. 2453-2467. DOI:10.1109/TMTT.2008.2004894

[29] Y. Cao, L. Simonovich, and Q. J. Zhang, 2009. A broadband and parametric model of differential via holes using space-mapping neural network, IEEE Microwave and Wireless Components Lett., vol. 19, pp.533-535. DOI:10.1109/LMWC.2009.202704 $\underline{8}$

[30] J. Rayas-Sanchez and Q. J. Zhang, 2012. On knowledge-based neural networks and neuro-space mapping, in IEEE Microwave Symposium Digest (MTT), pp. 1-3, 17-22 June, Montreal, QC, Canada. DOI: $10.1109 / M W S Y M .2012 .62583$ $\underline{54}$ 
M. Simsek / Knowledge Embedded Automatic Model Generation in Microwave Design Using Knowledge Based Artificial Neural Networks

[31] V. K. Devabhaktuni, M. C. E. Yagoub, and Q. J. Zhang, 2001. A robust algorithm for automatic development of neural-network models for microwave applications, IEEE Transactions on Microwave Theory and Techniques, vol. 49, no. 12, pp. 2282-2291. DOI: $\underline{10.1109 / 22.971611}$

[32] V. K. Devabhaktuni, B. Chattaraj, M. C. E. Yagoub, and Q. J. Zhang, 2003. Advanced microwave modeling framework exploiting automatic model generation, knowledge neural networks, and space mapping, IEEE Trans. Microwave Theory and Tech., vol. 51, no. 7, pp. 1822-1833.

DOI: 10.1109/TMTT.2003.814318

[33] ADS Momentum, 2014. Agilent Technologies, Palo, Alto, CA. 